\title{
HÁBITOS DE VIDA DOS TRILOBITAS DAS FORMAÇÕES MAECURU E ERERÊ, DEVONIANO DA BACIA DO AMAZONAS, BRASIL
}

\author{
CLEBER FERNANDES DA SILVA \\ Depto. de Geologia, UFRJ, Av. Brigadeiro Trompowsky, s/n, Cidade Universitária, Ilha do Fundão, 21949-900, \\ Rio de Janeiro, RJ, Brasil.cleberfer@uol.com.br
}

VERA MARIA MEDINA DA FONSECA

Depto. de Geologia e Paleontologia, MN/UFRJ, Quinta da Boa Vista, São Cristóvão, 20940-040, Rio de Janeiro, RJ, Brasil. vmedina@acd.ufrj.br

\begin{abstract}
RESUMO - Oito das 14 espécies de trilobitas identificadas nas formações Maecuru e Ererê (Pará), foram submetidas a uma análise morfofuncional e agrupadas em três morfotipos. O morfotipo I caracteriza-se pelo perfil achatado, céfalo pouco inflado, grande área pré-glabelar em forma de pá; glabela e lobos glabelares frontal e laterais pouco inflados, sulcos glabelares rasos e olhos pequenos. O morfotipo II caracteriza-se pelo céfalo moderadamente inflado, podendo possuir um pequeno processo mediano frontal; glabela moderadamente inflada, lobo glabelar frontal moderadamente inflado e expandido anteriormente; lobos glabelares laterais moderadamente inflados e sulcos glabelares laterais mais profundos; meio-anéis de articulação bem desenvolvidos, hipostômio fixo contérmino e olhos médios a grandes. O morfotipo III caracteriza-se pela glabela e lobo glabelar frontal bastante inflados, lobos glabelares laterais moderadamente inflados, sulcos glabelares moderadamente rasos e olhos de tamanho médio a grande. Todas as espécies apresentam características de hábito alimentar predador/necrófago. As dos morfotipos I e II possuiriam hábito predominantemente epibentônico com adaptações para escavação, mais desenvolvidas no morfotipo I, usadas para alimentação ou proteção. A cavidade estomacal bem desenvolvida das espécies do morfotipo III, sugere que tenham sido predadoras mais ávidas do que as demais espécies.
\end{abstract}

Palavras-chave: trilobita, paleoautoecologia, Devoniano, Formação Maecuru, Formação Ererê, bacia do Amazonas.

ABSTRACT - LIFE HABITS OF TRILOBITES FROM THE MAECURU AND ERERE FORMATIONS (DEVONIAN), AMAZON BASIN, BRAZIL. Eight of the 14 trilobite species identified in deposits of the Maecuru and Ererê formations (Middle Devonian, Amazonas basin, Pará State, Brazil) were submitted to a functional analysis and were grouped into three morphotypes. Morphotype I presents a flattened profile, a slightly inflated cephalon with a large preglabellar shovel-shaped area, glabella, frontal and lateral glabellar lobes slightly inflated, shallow glabellar furrows and small eyes. The morphotype II is characterized by a moderately inflated cephalon, which may present a small median frontal process; glabella moderately inflated, glabellar frontal lobe moderately inflated and anteriorly expanded; glabellar lateral lobes moderately inflated and deeper glabellar furrows; half-rings well developed, a fixed conterminant hypostome and medium to large sized eyes. The morphotype III presents strongly inflated glabela and glabelar frontal lobes, glabellar lateral lobes moderately inflated, glabellar furrows moderately shallow and eyes ranging from medium to large sizes. All the species show predator/scavenger feeding habits. Species of the morphotypes I and II have a predominantly epibenthic mode of life, presenting adaptations to burrowing. Such adaptations were probably either used for feeding or protection. The well-developed stomach cavity in the trilobites referred to the morphotype III suggests they had been more voracious predators than those belonging to the other morphotypes.

Key-words: trilobite, paleoautoecology, Devonian, Maecuru Formation, Ererê Formation, Amazon Basin. 


\section{INTRODUÇÃO}

Os trilobitas foram os artrópodes marinhos mais diversificados e abundantes do Paleozóico. Já no início de sua evolução apresentavam hábitos alimentares diversificados, que os tornaram capazes de explorar diferentes tipos de nichos, sendo uma das explicações para o seu grande sucesso. Um dos hábitos alimentares mais primitivos seria o epibentônico predador (Jensen, 1990), porém, hábitos alimentares detritívoros já se faziam presentes no Cambriano (Fortey \& Owens, 1999). Ao longo de sua evolução, os trilobitas desenvolveram
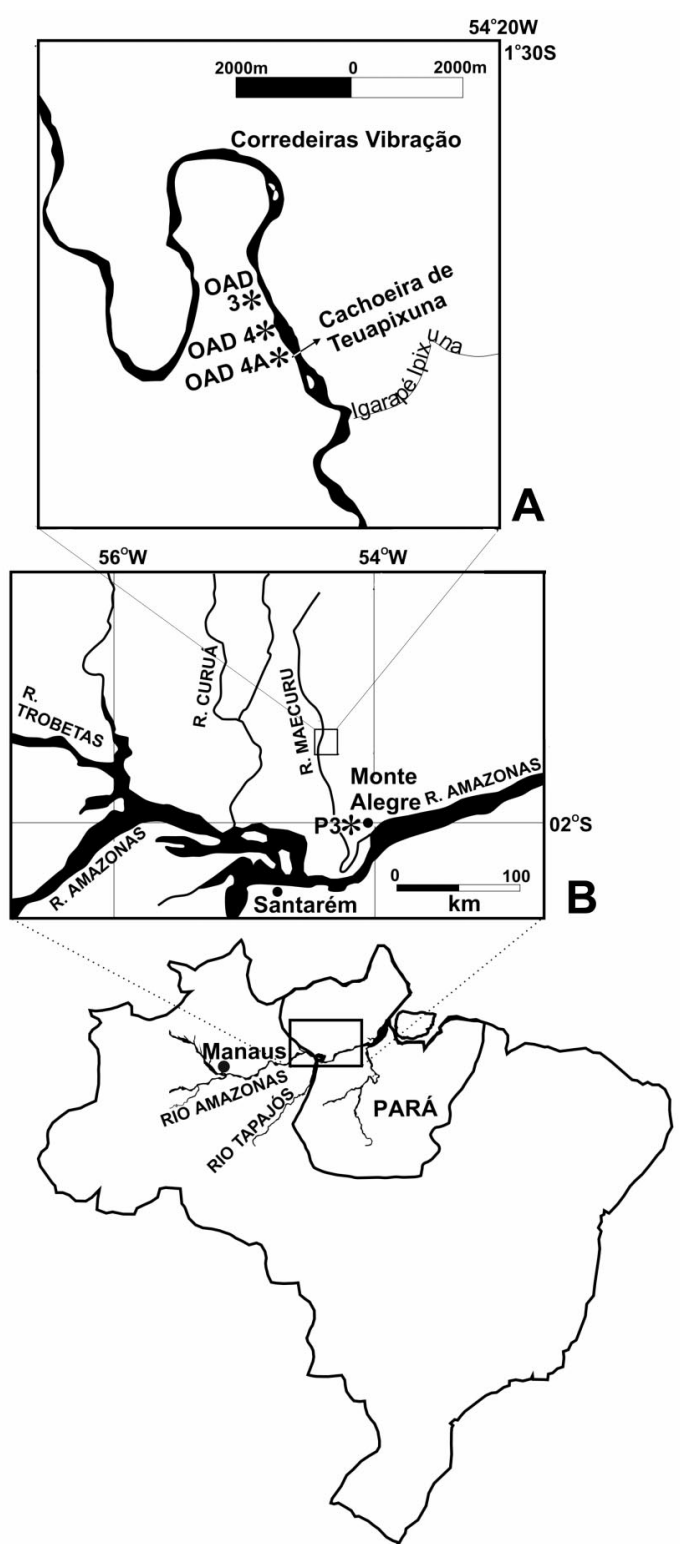

Figura 1. Mapa de localização dos afloramentos fossilíferos (bacia do Amazonas). A, rio Maecuru e B, região de Monte Alegre (modificado de Machado et al., 1996).

Figure 1. Location map of the fossiliferous outcrops (Amazon Basin). A, Maecuru river and B, Monte Alegre region (modified from Machado et al., 1996). outros tipos de hábitos, tornando-se cada vez mais especializados. Há evidências de trilobitas escavadores, pelágicos, filtradores e planctônicos (Fortey \& Owens, 1999).

$\mathrm{O}$ estudo dos trilobitas vem sendo abordado sob diferentes enfoques há pelo menos três décadas (para histórico, ver Adrain \& Westrop, 1999). No Brasil, estudos paleontológicos recentes tratando de trilobitas da bacia do Paraná foram feitos por Ghilardi, 2004. Entretanto, pouco foi acrescentado ao conhecimento dos trilobitas da bacia do Amazonas desde os trabalhos pioneiros de Hartt \& Rathbun (1875), Clarke (1895) e Katzer (1903).

A partir da análise morfofuncional, são apresentados aqui os hábitos de vida dos trilobitas das formações Maecuru e Ererê, com ênfase em seus hábitos alimentares.

\section{MATERIAL E MÉTODOS}

O material estudado constitui-se de 53 amostras coletadas pela Comissão Geológica do Império, Expedição Orville A. Derby e pelo Serviço Geológico e Mineralógico do Brasil, depositadas em três instituições: Departamento de Geologia e Paleontologia do Museu Nacional/UFRJ (MN-I), Instituto de Geociências da Universidade Federal do Rio de Janeiro (UFRJ-DG-Tr) e Museu de Ciências da Terra do Departamento Nacional de Produção Mineral (MCTer-I).

A terminologia utilizada segue Harrington (1959), Clarkson (1998) e Gon III (2003) e a classificação das espécies, Clarke (1895), Eldredge \& Ormiston (1979), Lieberman et al. (1991) e Lieberman (1993). Eldredge \& Ormiston (1979) sugeriram que as espécies Dalmanites maecurua, D. galeus e D. tumilobus, descritas originalmente por Clarke (1895), pertenceriam a novos três gêneros afins aos gêneros Fenestraps, Vogesina e Malvinella, sem, contudo, descrevê-los. Assim, neste trabalho, optou-se por empregar para essas espécies os nomes originais de Clarke (1895).

Das 14 espécies presentes nas formações Maecuru e Ererê, oito, que possuíam céfalos e hipostômios, foram submetidas a análises morfofuncionais para inferir os hábitos alimentares. Estas análises basearam-se no trabalho de Fortey \& Owens (1999), que identificaram cinco tipos de hábitos alimentares nos trilobitas (predador/necrófago, detritívoro, suspensívoro, filtrador e parasita), fundamentados na morfologia externa dos céfalos e hipostômios dos mesmos.

\section{LOCALIZAÇÃO GEOGRÁFICA DOS AFLORAMENTOS}

O material da Formação Maecuru aqui estudado foi coletado em diferentes localidades ao longo do rio Maecuru, no Estado do Pará, pela Comissão Geológica do Império, em 1876, e pela Expedição Orville A. Derby (OAD), realizada pela Petrobras em 1986. A localidade de coleta da Comissão Geológica do Império é citada apenas como "rio Maecuru". Localizações mais precisas de afloramentos fossilíferos de- 
vonianos do mesmo rio foram obtidas, mais tarde, pela Expedição Orville A. Derby, descritas a seguir (Figura 1):

OAD 3-Margem direita do rio Maecuru, a cerca de $900 \mathrm{~m}$ da cachoeira de Teuapixuna ou Alagação e a aproximadamente $2,1 \mathrm{~km}$ a montante do igarapé Ipixuna, Estado do Pará.

OAD 4-Margem direita do rio Maecuru, a cerca de $400 \mathrm{~m}$ a montante da cachoeira de Teuapixuna ou Alagação e 1,5 km a montante do igarapé Ipixuna, Estado do Pará.

OAD 4A-Cachoeira de Teuapixuna ou Alagação, a cerca de $1,2 \mathrm{~km}$ a montante do igarapé Ipixuna no rio Maecuru, Estado do Pará.

Foi observado que o conteúdo fossilífero e a litologia das amostras coletadas pela Comissão Geológica do Império são semelhantes aos dos pontos OAD 4 e 4A, enquanto aquelas estudadas por Katzer (1903) assemelham-se às do ponto OAD 3.

O material da Formação Ererê coletado pela Comissão Geológica do Império e o material estudado por Katzer (1903) provêm de uma localidade citada apenas como "Ererê" no Estado do Pará. Provavelmente, corresponde à área tipo da Formação Ererê, localizada na planície de Ererê, a nordeste e a leste da serra de Ererê, no município de Monte Alegre (Oliveira \& Leonardos, 1978). Outro ponto de coleta da Formação Ererê foi a sondagem n ${ }^{\circ} 84$ do Serviço Geológico e Mineralógico do Brasil (sonda de Itauajuri) na região de Monte Alegre, no campo da Malhada Grande (Oliveira \& Barbosa, 1940), distante $2.350 \mathrm{~m}$ do furo 78, a cerca de 11 $\mathrm{km}$ do trapiche da cidade de Monte Alegre no Estado do Pará (Guimarães, 1936) (Figura 1).

\section{CONTEXTO GEOLÓGICO E TAFONÔMICO}

Os trilobitas devonianos da bacia do Amazonas ocorrem no Grupo Urupadi, subdividido, da base para o topo, nas formações Maecuru (membros Jatapu e Lontra) e Ererê (Figura 2), cuja deposição iniciou-se no Lochkoviano (Eodevoniano), encerrando-se no Mesodevoniano (Givetiano) (Melo \& Loboziak, 2001; Loboziak \& Melo, 2002).

Os trilobitas da Formação Maecuru ocorrem em tempestitos, constituídos por arenitos marinhos finos a grossos, com estratificação cruzada tipo hummocky, de idade eifeliana, presentes em seu topo (Membro Lontra) (Melo, 1988; Melo \& Loboziak, 2001; Loboziak \& Melo, 2002). Constituem juntamente com braquiópodes, moluscos, briozoários, crinóides, colunariídeos e tentaculitídeos - a tafocenose de macroinvertebrados marinhos mais diversificada do Devoniano brasileiro (Fonseca, 2001). O material estudado está preservado em blocos coletados ao longo das margens do rio Maecuru, no Pará. Não existem, entretanto, informações a respeito de suas posições estratigráficas. Machado et al. (1996) constataram a ocorrência de diferentes tafocenoses em blo-

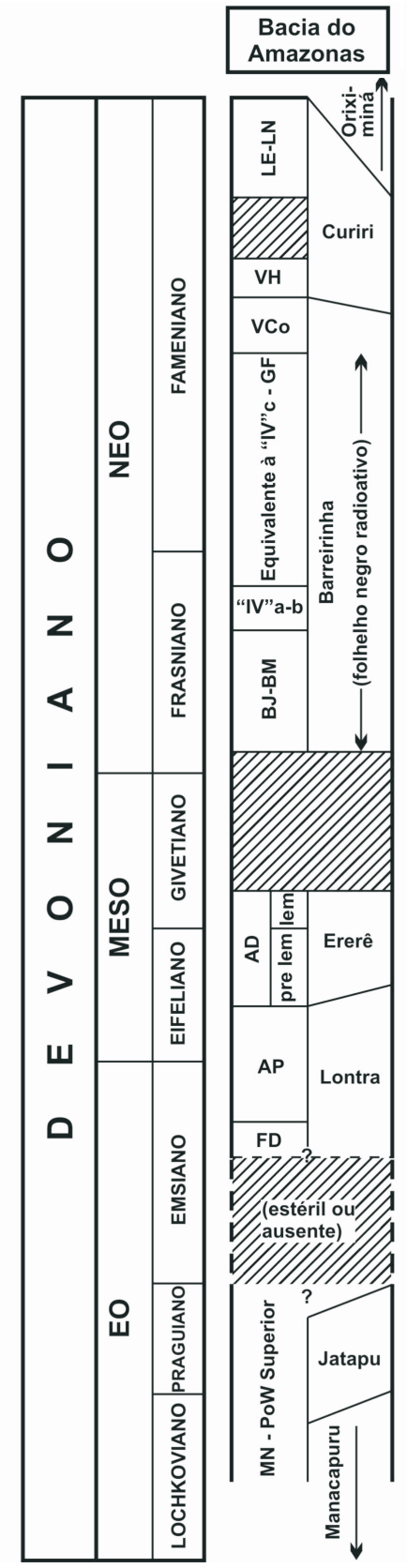

Figura 2. Biozonas de miósporos do Devoniano da bacia do Amazonas e correlação com as unidades litoestratigráficas regionais (modificado de Loboziak \& Melo, 2002).

Figure 2. Miospore zonal schemes for the Devonian of the Amazon Basin and their relationships with regional lithoestratigraphic units (modified from Loboziak \& Melo, 2002).

cos de arenito coletados em cinco pontos ao longo do rio Maecuru, três dos quais (pontos OAD 3, OAD 4 e OAD 4A) são localidades de coleta dos restos dos trilobitas estudados. $\mathrm{Na}$ tafocenose do ponto OAD 3 foi identificada apenas uma espécie de trilobita, "Palpebrops" goeldii (Katzer, 1903). Nas amostras de mão examinadas, os exoesqueletos de trilobitas encontram-se dissociados, associados a conchas de braquiópodes e discos de colunas de crinóides, constituindo uma concentração de bioclastos politípica, densamente empaco- 

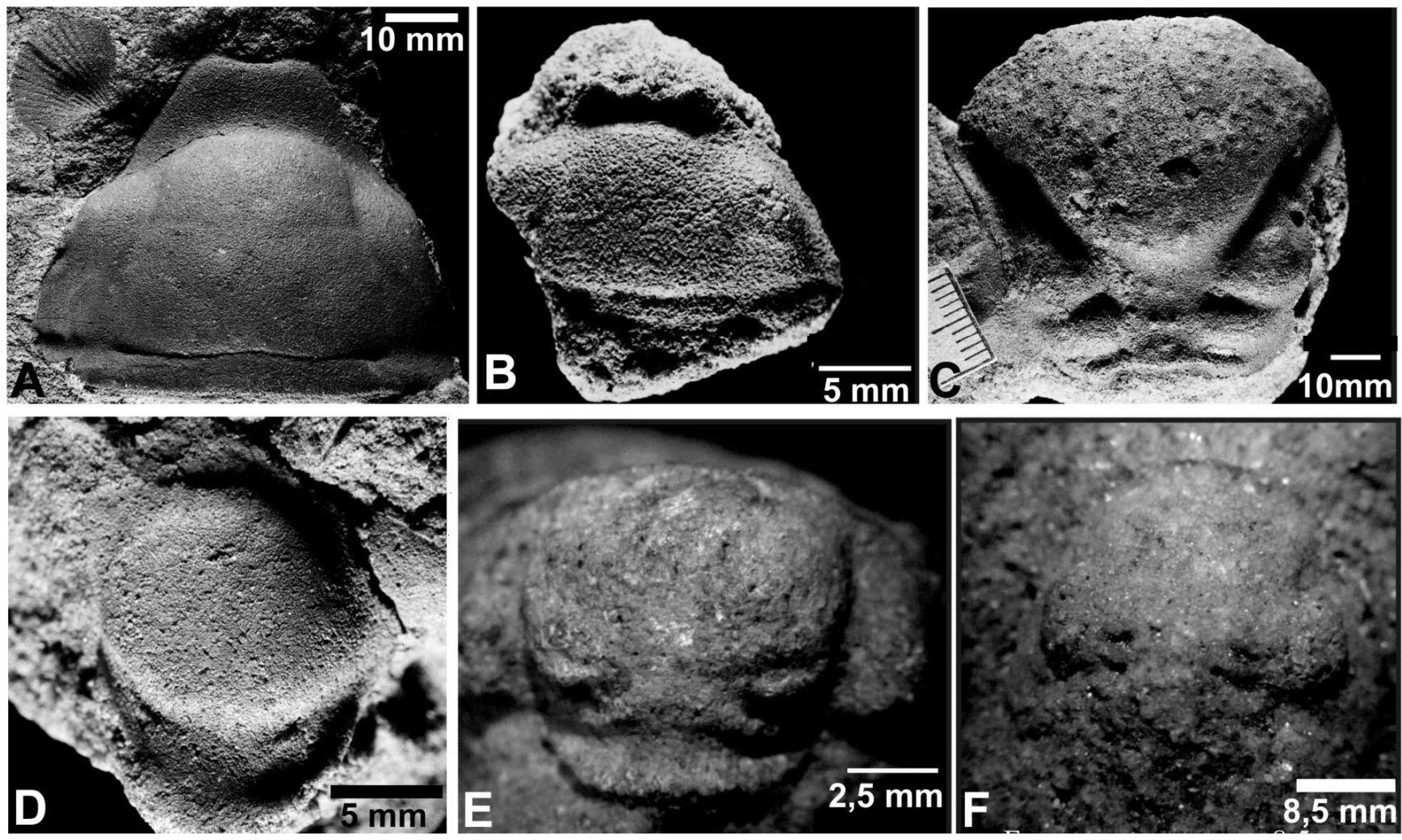

Figuras 3. Homalonotus derbyi: A, molde interno de um céfalo quase completo (MN 3372-I); B, molde interno de um pequeno céfalo fragmentado (MN 3371-I); C-D, Dalmanites maecurua: C, molde interno de uma glabela fragmentada (MN 3383-I); D, Hipostômio fragmentado e desgastado (MN 3384-I); E, Dalmanites galeus: molde interno de céfalo completo desgastado (MN 3390-I); F, Phacopina brasiliensis: molde interno de céfalo completo desgastado (MN 3375-I).

Figures 3. Homalonotus derbyi: A, internal mold of almost complete cephalon (MN 3372-I); B. internal mold of fragmented tiny cephalon (MN 3371-I); C-D, Dalmanites maecurua: C, internal mold of a fragmented glabella (MN 3383-I); D. Fragmented and worn-out hypostome (MN 3384-I); E, Dalmanites galeus: internal mold of a worn-out cephalon (MN 3390-I); (F). Phacopina brasiliensis: internal mold of a worn-out cephaIon (MN 3375-I).

tada, na qual os bioclastos encontram-se reorientados de forma caótica, fragmentados, mas pouco desgastados, sugerindo pouco transporte, e uma provável origem parautóctone. As tafocenoses dos pontos OAD 4, OAD 4A e os exemplares da coleção feita no rio Maecuru pela Comissão Geológica do Império apresentam todas as outras espécies de trilobitas da Formação Maecuru. No ponto OAD 4, ocorrem céfalos e/ou glabelas das espécies "Malvinella" australis (Clarke, 1895) $e$ Dalmanites, tumilobus Clarke, 1895 e no ponto OAD 4a uma glabela de Dalmanites maecurua Clarke, 1895. Todas essas espécies, com exceção de "Palpebrops" goeldii, além das espécies Homalonotus derbyi Clarke, 1895, Dalmanites galeus Clarke, 1895 e Phacopina brasiliensis (Clarke, 1895), foram também coletadas no rio Maecuru pela Comissão Geológica do Império. Não é possível saber se esses fósseis são procedentes de duas ou três localidades diferentes e de um único ou de diferentes níveis estratigráficos. De uma maneira geral, as tafocenoses dessas localidades são politípicas, exibem restos fósseis desarticulados, fracamente empacotados a dispersos, apresentando ou não fragmentação, alguns com leves marcas de abrasão. $O$ fato de haver diferentes graus de preservação pode indicar eventos deposicionais diferentes, mistura temporal e espacial, com associações de elementos parautóctones a alóctones.

A Formação Ererê consiste de siltitos micáceos intercalados por folhelhos e arenitos de granulação fina, depositados em ambiente deltaico a nerítico da metade do Eifeliano a metade do Givetiano (Melo, 1988; Melo \& Loboziak, 2001; Loboziak \& Melo, 2002). O registro fossilífero desta formação ocorre em especial na base da mesma, em depósitos de tempestades (Melo, 1988; Cunha et al., 1994). Associados aos trilobitas são encontrados fósseis de braquiópodes, moluscos, ostracodes, escolecodontes e tentaculitídeos (Melo, 1988; Fonseca, 2001). Melo (1988) observou uma queda na diversidade faunística da Formação Ererê em relação à fauna da Formação Maecuru. Esta queda é facilmente observada quando se compara a diversidade específica dos trilobitas presentes na Formação Maecuru (14 espécies) com aquela 

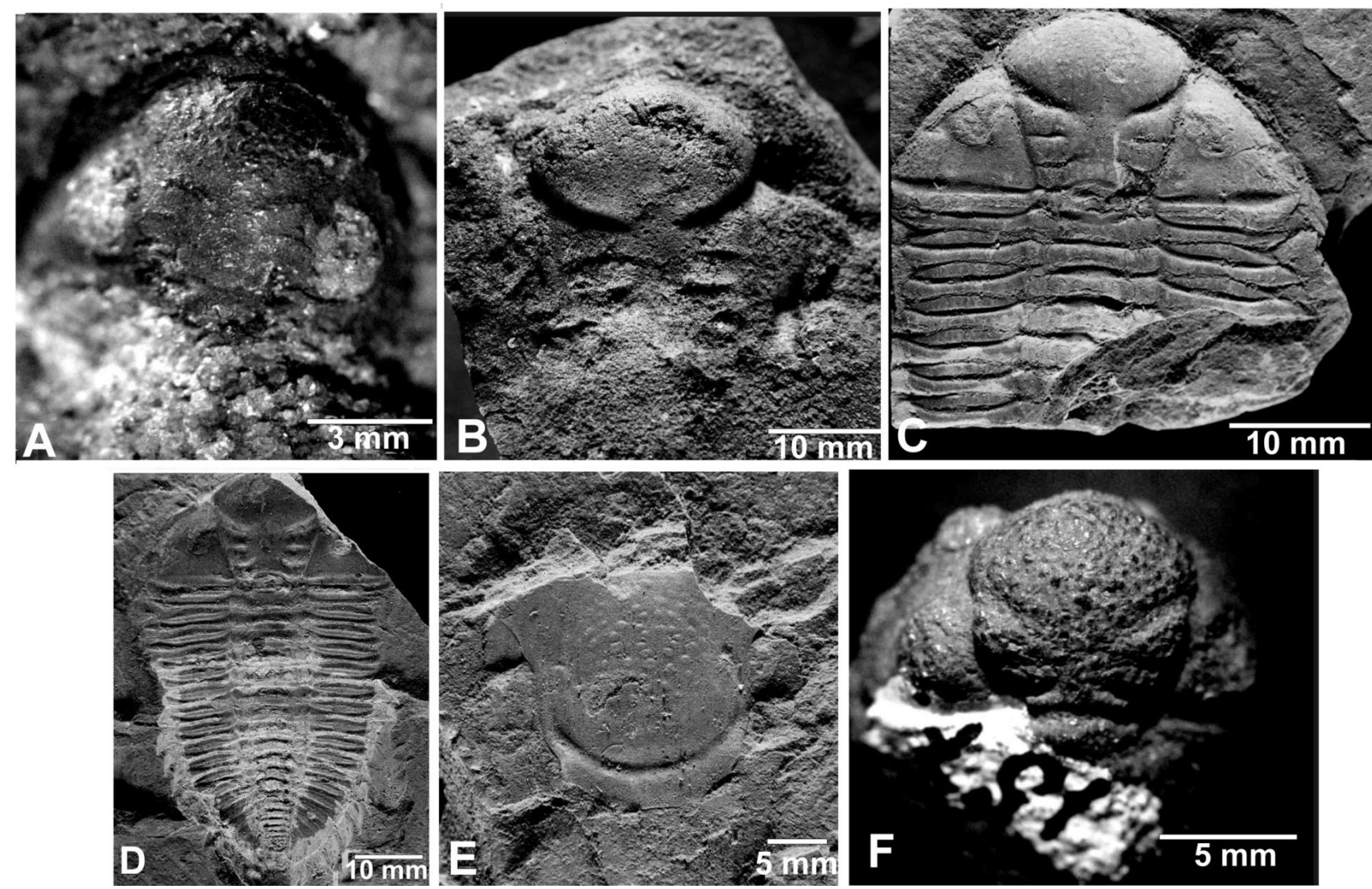

Figure 4. A, Phacopina brasiliensis: molde interno de céfalo quase completo (MN 3376-I); B-E, Eldredgeia paituna: B, glabela fragmentada preservada em arenito (MN 3395-I); C-E, preservados em folhelho; C, molde interno de céfalo e alguns segmentos toráxicos (MCTer 2799-I); D. molde externo de um indivíduo inteiro (MCTer 2807-I); E, hipostômio (MCTer 2791-I); F,“Malvinella” australis: molde interno de um céfalo (MN 3389-I).

Figura 4. A, Phacopina brasiliensis: internal mold of an almost complete cephalon (MN 3376-I); B-E, Eldredgeia paituna: B, fragmented glabella preserved in sandstone (MN 3395-I); C-E, preserved in shale: C, internal mold of a cephalon and a few thoracic segments (MCTer 2799-I); D, external mold of a complete specimen (MCTer 2807-I); E, hypostome (MCTer 2791-I); F, "Malvinella" australis: internal mold of cephalon (MN 3389-I).

da Formação Ererê (três espécies), das quais apenas uma, Eldredgeia paituna (Hartt \& Rathbun,1875), foi aqui estudada por apresentar céfalo e hipostômio. Estes estão preservados em dois tipos de sedimento, arenito e folhelho, procedentes respectivamente da planície de Ererê e da sondagem n ${ }^{\circ} 84$ do Serviço Geológico e Mineralógico, na região de Monte Alegre. No arenito foram encontradas apenas glabelas com marcas de abrasão, sugerindo transporte, evidenciado pelo desgaste da região do lobo frontal e fragmentação da margem posterior, onde houve a perda do anel occipital. Os fósseis preservados no folhelho aparentam ter sofrido muito pouco transporte ou pequena bioturbação pós-morte, fato evidenciado pela presença no mesmo sítio de indivíduos inteiros, céfalos e pigídios bem preservados, além de hipostômios e segmentos toráxicos. As observações acima sugerem que a associação do arenito poderia ser parautóctone ou alóctone e a do folhelho autóctone ou parautóctone.

\section{RESULTADOS}

A partir de análise morfofuncional, foram reconhecidos 3 morfotipos:

Morfotipo I. Representado pela espécie Homalonotus derbyi (MN 3370-I a MN 3373-I, Figura 3A-B), é caracterizado por possuir perfil achatado, céfalo pouco inflado (sem levar em conta possíveis achatamentos provocados por eventos pós-morte), com uma grande área pré-glabelar em forma de pá; glabela pouco inflada, no mesmo plano das regiões genais; lobos glabelares laterais pouco inflados, com sulcos glabelares laterais muito rasos; lobo glabelar frontal diferen- 

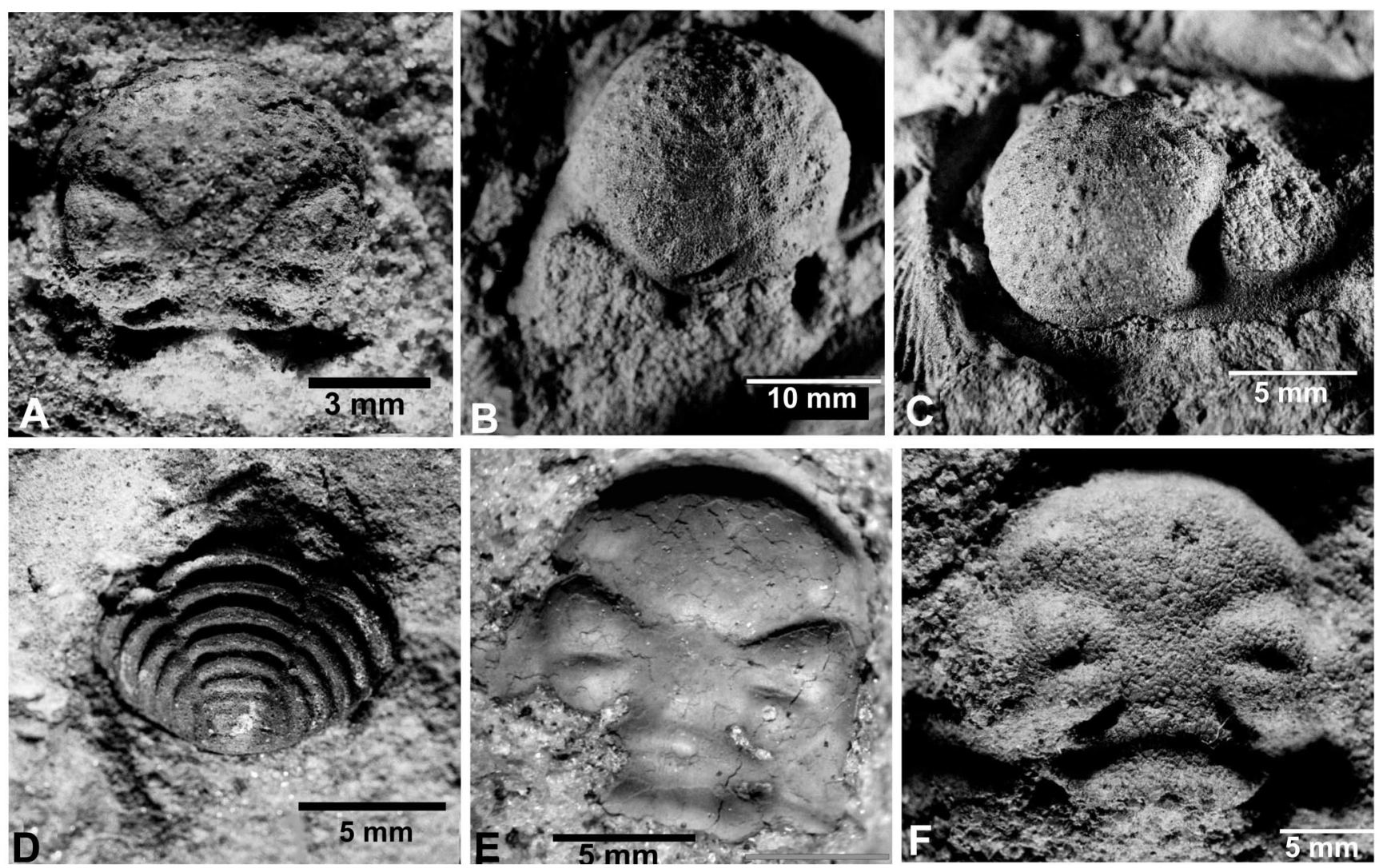

Figura 5. A, "Malvinella" australis: molde interno de glabela (UFRJ-DG 114-Tr); B-D, "Palpebrops" goeldii: B-C, molde interno de cranidium parcialmente preservado, B, vista dorsal; C, vista lateral oblíqua (UFRJ-DG 112-Tr); D, pigídio (UFRJ-DG 118-Tr); E-F, Dalmanites tumilobus: E, molde interno de glabela com processo mediano frontal (UFRJ-DG 116-Tr); F. molde interno de uma glabela pouco desgastada (MN 3391-I). Figure 5. A, "Malvinella" australis: internal mold of glabella (UFRJ-DG 114-Tr); B-D, "Palpebrops" goeldii: B-C, internal mold of cranidium; B, dosal view; C, antero-lateral view (UFRJ 112-Tr); D, pigidium (UFRJ 118-Tr); E-F, Dalmanites tumilobus: E, internal mold of glabella, showing a frontal mediam process (UFRJ-DG 116-Tr); F, internal mold of a litlle worn-out glabella (MN 3391-I).

ciado, porém, pouco inflado; olhos pequenos, sobre grandes lobos palpebrais, convexos.

Morfotipo II. Pertencem a este morfotipo Dalmanites maecurua (MN 3381-I a MN 3388-I, UFRJ-DG 115-Tr, Figura 3C-D), D. galeus (MN 3390-I, Figura 3E), Phacopina brasiliensis (MN 3375-I, MN 3376-I, Figuras 3F, 4A); e D. tumilobus (MN 3391-I a MN 3393-I, UFRJ-DG 116-Tr; Figura 5E-F) da Formação Maecuru e Eldredgeia paituna (MN 3394-I, MN 3395-I, MCTer 2791-I a MCTer 2793-I, MCTer 2799-I, MCTer 2800-I, MCTer 2802-I a MCTer 2804-I, MCTer 2807-I, Figura 4B-E) da Formação Ererê. Apresentam céfalos moderadamente inflados, podendo possuir um pequeno processo mediano frontal; glabela moderadamente inflada, em um plano um pouco acima das regiões genais; sulcos glabelares laterais mais profundos do que os da espécie do morfotipo I, possibilitando uma boa distinção de todos os lobos glabelares laterais, que são moderadamente inflados; lobo glabelar frontal bastante diferenciado da região glabelar posterior, expandido anteriormente, mas não ultrapassando a borda cefálica, e moderadamente inflado; olhos médios a grandes, com uma boa superfície visual; meio-anéis de articulação bem desenvolvidos e hipostômio fixo contérmino. Algumas formas apresentam tubérculos sobre a glabela.

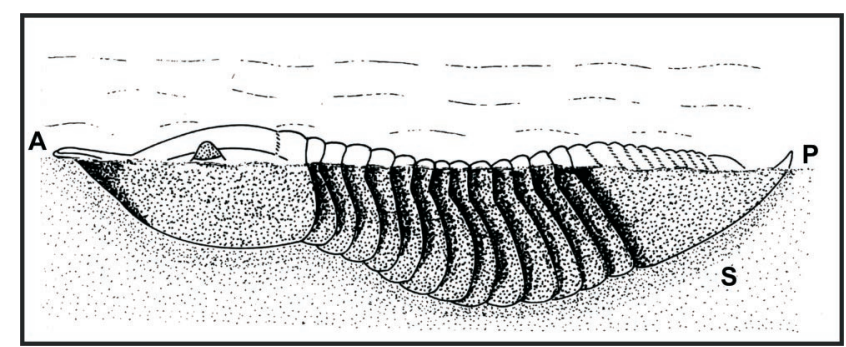

Figura 6. Possível postura de Trimerus delphinocephalus em escavação rasa no sedimento inconsolidado (mod. dWhittington, 1993). $A=$ anterior; $P=$ posterior; $S=$ sedimento.

Figure 6. Trimerus delphinocephalus possible attitude in shallow excavation into loose sediment (modified from Whittington, 1993). $\mathrm{A}=$ anterior $\mathrm{P}=$ posterior $; \mathrm{S}=$ sediment.

Morfotipo III. Pertencem a este morfotipo as espécies "Malvinella" australis (MN 3389-I, UFRJ-DG 114-Tr, Fi- 
guras 4F e 5A) e "Palpebrops" goeldii (UFRJ-DG 111-Tr, UFRJ-DG 112-Tr, UFRJ-DG 118-Tr, Figura 5B-D). Apresentam glabela bastante inflada, localizada bem acima do plano das regiões genais; sulcos glabelares moderadamente rasos, dificultando em alguns casos a distinção dos limites dos lobos glabelares laterais; lobos glabelares laterais todos no mesmo plano, moderadamente inflados; lobo glabelar frontal bastante inflado, inclinando-se abruptamente para a margem cefálica anterior.

\section{DISCUSSÃO}

\section{Morfotipo I}

A glabela pouco inflada e os lobos e sulcos glabelares laterais pouco visíveis (definidos) dessa forma indicam que sua área estomacal não seria extensa. Isso sugere que o trilobita não ingeriria um grande volume de comida, característica que, segundo Fortey \& Owens (1999), caracterizaria um hábito trófico detritívoro, pois o animal engoliria partículas pequenas. Por outro lado, o lobo glabelar frontal bem diferenciado, embora pouco inflado, indica que a área estomacal deveria ser maior do que se esperaria de um trilobita detritívoro, estando mais próximo a um trilobita predador/necrófago.

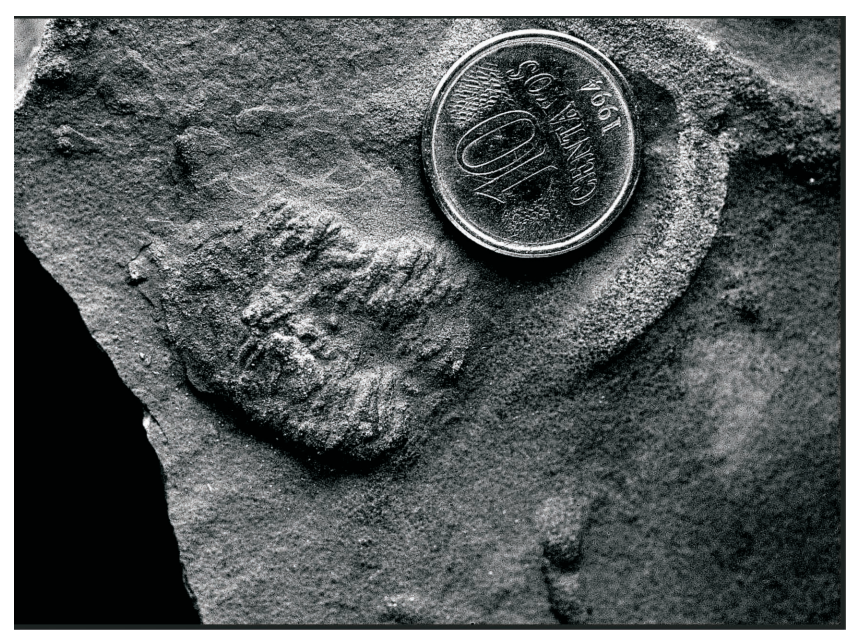

Figura 7. Rusophycus da Formação Pimenteira (interceptando um rastro de verme?)

Figure 7: Rusophycus of the Pimenteira Formation (intercepting a worm track?)

O perfil e a área pré-glabelar achatados, a última em forma de pá, e a ausência de tubérculos na superfície dorsal do céfalo, diminuiriam o atrito do trilobita com o sedimento, indicando uma possível adaptação à escavação. Esta suposição foi feita por Whittington (1993) para a espécie Trimerus delphinocephalus Green, 1832, que, morfologicamente, é muito similar a Homalonotus derbyi, tendo a capacidade de escavar buracos rasos, ficando com parte do céfalo e pigídio a mostra (Figura 6). Outro fato que reforça um possível hábito epibentônico com adaptações para escavação para a espé- cie estudada é a suposição de que Fexicalymene aff. granulo$s a$ (Foerste, 1909), um calimenídeo de morfologia semelhante à da espécie estudada, possui este tipo de hábito (Hughes \& Cooper, 1999) e que sua musculatura é similar a outros calimenídeos, incluindo Trimerus (Whittington, 1993).

Em relação ao hipostômio, não há nenhuma amostra da bacia do Amazonas com essa estrutura preservada que pudesse ter sido relacionada à espécie do morfotipo I. Fortey \& Owens (1999) observaram projeções (forks) nos hipostômios de calimenídeos e homalonotídeos, características de um hábito alimentar predador/necrófago.

\section{Morfotipo II}

O lobo glabelar frontal não expandido para além da borda cefálica anterior, porém, chegando no limite da mesma, indica um hipostômio do tipo fixo contérmino, firmemente preso à dobra cefálica, conforme o modelo de Fortey \& Owens (1999). Esta afirmação é reforçada pela presença de asas anteriores de fixação bem desenvolvidas no hipostômio de Eldredgeia paituna, característica típica de hipostômios fixos. Essas mesmas asas anteriores de fixação também podem ser observadas no hipostômio atribuído à espécie Dalmanites maecurua. A margem posterior do hipostômio de E. paituna apresenta dois pares de pequenas projeções em forma de espinho, que são estruturas presentes em trilobitas de hábito alimentar predador/necrófago (Fortey \& Owens, 1999). Embora o hipostômio de D. maecurua esteja com sua borda posterior desgastada, assemelha-se muito (assim como a morfologia da glabela) ao hipostômio da espécie boliviana Franchovichia francovichi Branisa, 1965 (Branisa, 1965; plates $14 \mathrm{e} 73: 5)$, que apresenta estruturas semelhantes à encontrada em E. paituna, reforçando a idéia de um hábito alimentar predador/necrófago.

A forma moderadamente convexa da glabela, e a presença de pequenos tubérculos indicam que as espécies do morfotipo II não seriam escavadoras tão rápidas como a do morfotipo I. Este fato, entretanto, não descarta a possibilidade de escavarem o sedimento para capturar alguma presa ou mesmo se proteger. Silva (2001), estudando os invertebrados fossilizados da Formação Cabeças (Devoniano da bacia do Parnaíba), assinalou um hábito predominantemente epibentônico para a espécie Metacryphaeus meloi Carvalho et al., 1997, baseando-se também na morfologia do céfalo e hipostômio da mesma. A espécie da Formação Cabeças é muito similar à E. paituna. Provavelmente, as espécies do morfotipo II rastejariam sobre os sedimentos de fundo a caça de pequenos animais de corpo mole, ou alimentando-se de carne morta. Icnofósseis atribuíveis a trilobitas ainda não foram encontrados nas formações Maecuru e Ererê. Contudo, depósitos devonianos da bacia do Parnaíba (Formação Pimenteira), localizada a leste da bacia do Amazonas, contêm Rusophycus Hall, 1852. Alguns desses icnofósseis, segundo Fortey \& Owens (1999), podem estar relacionados à atividade de predação. Às vezes, interceptam, no mesmo plano, rastros de 
vermes, onde visivelmente ocorre um aprofundamento por parte dos últimos, indicando uma tentativa de fuga. O espécime de Rusophycus da Formação Pimenteira (Figura 7) poderia ter sido produzido por algum trilobita semelhante aos das espécies do morfotipo II, visto que as faunas das duas bacias são muito semelhantes (Machado, 1990; Fonseca, 2001).

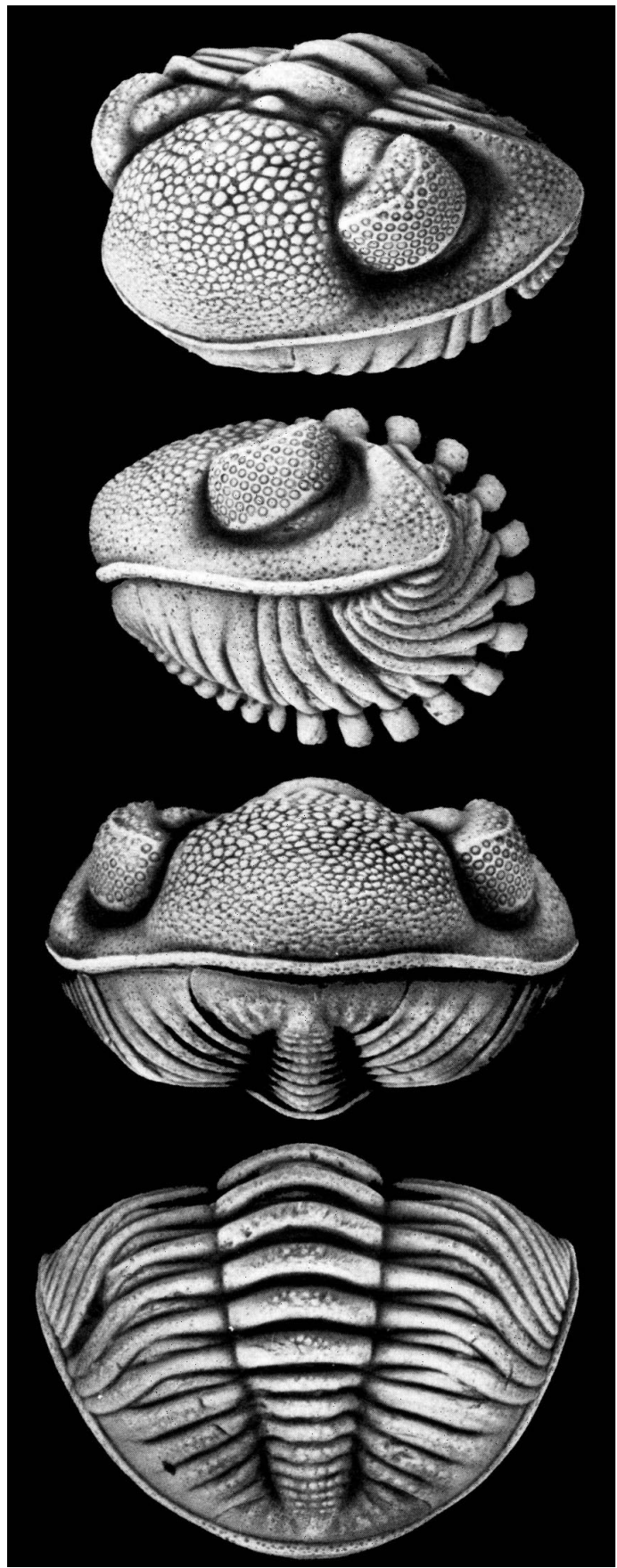

Figura 8. Phacops rana. Espécime enrolado para se proteger (modificado de Kesling \& Chilman, 1975).

Figure 8. Phacops rana. Specimen enrolled for protection (modified from Kesling \& Chilman, 1975).
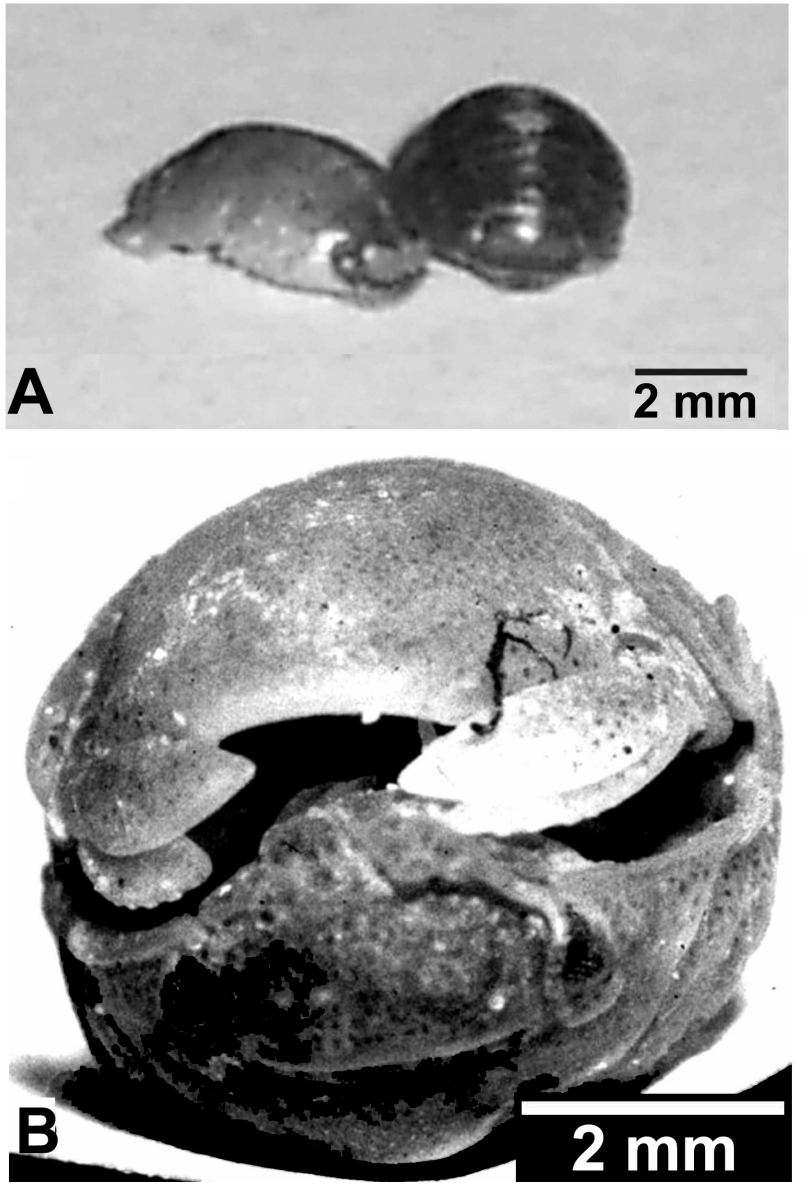

Figura 9. Sphaeroma serratum: A, em posição normal; B, espécime enrolado para se defender contra predadores ou distúrbios ambientais.

Figure 9. Sphaeroma serratum: A, specimem extended; B, specimen enrolled for protection against predators or environmental disturbances.

A presença de meio-anéis (half rings) de articulação bem desenvolvidos em E. paituna aponta para uma capacidade de enrolamento desses trilobitas. Esta ação não é incomum em facopídeos, como mostrado por Kesling \& Chilman (1975) em Phacops rana (Green, 1822) (Figura 8) e no gênero Trimerus Green, 1832 (Whittington, 1993). O mesmo padrão de enrolamento pode ser observado no crustáceo atual Sphaeroma serratum (Fabricius, 1787) (Figura 9). O enrolamento poderia ser causado por algum distúrbio ambiental ou como uma forma de proteção dos predadores (Brito et al., 2000, Domingues, 2003).

\section{Morfotipo III}

O hábito trófico dessas espécies provavelmente foi o mesmo reconhecido nos morfotipos anteriores. Sua glabela bastante inflada, em especial o lobo glabelar frontal, indica uma área estomacal considerável, maior do que as presentes nas espécies do morfotipos I e II. Esses animais provavelmente ingeririam uma quantidade de comida maior que 
aqueles referidos aos morfotipos anteriores, sendo predadores/necrófagos mais ávidos. Os olhos apresentam uma grande superfície ocular, posicionados látero-frontalmente, a uma altura que proporcionaria uma boa visão por sobre o escudo cefálico, o que favoreceria o hábito predador.

Nenhum hipostômio relacionado a essas espécies foi observado, contudo, as características morfológicas de suas glabelas indicariam hipostômio do tipo fixo. A observação do céfalo de "Malvinella" australis deixa a impressão de que o lobo glabelar frontal se estende para além da margem cefálica anterior. No entanto, ao ser observado lateralmente fica claro que o lobo glabelar frontal não ultrapassa a linha da margem anterior cefálica. Esses fatos nos levam a concluir que o hipostômio das duas espécies do morfotipo III, embora não ocorra um céfalo totalmente preservado de "Palpebrops" goeldii no material estudado, seria do tipo fixo contérmino, segundo o modelo de Fortey \& Owens (1999).

Provavelmente, seriam animais epibentônicos e se enquadrariam no modelo proposto por Batt (1995) para Phacops rana, que é considerado como móvel epibentônico. $\mathrm{O}$ mesmo morfotipo é apresentado nos esquemas de McKerrow (1981) sempre como epibentônico (Figura 10). Este modelo também é coerente para o morfotipo III, visto que sua glabela bastante inflada e os tubérculos grossos na superfície do céfalo aumentariam a área de contato com o se-

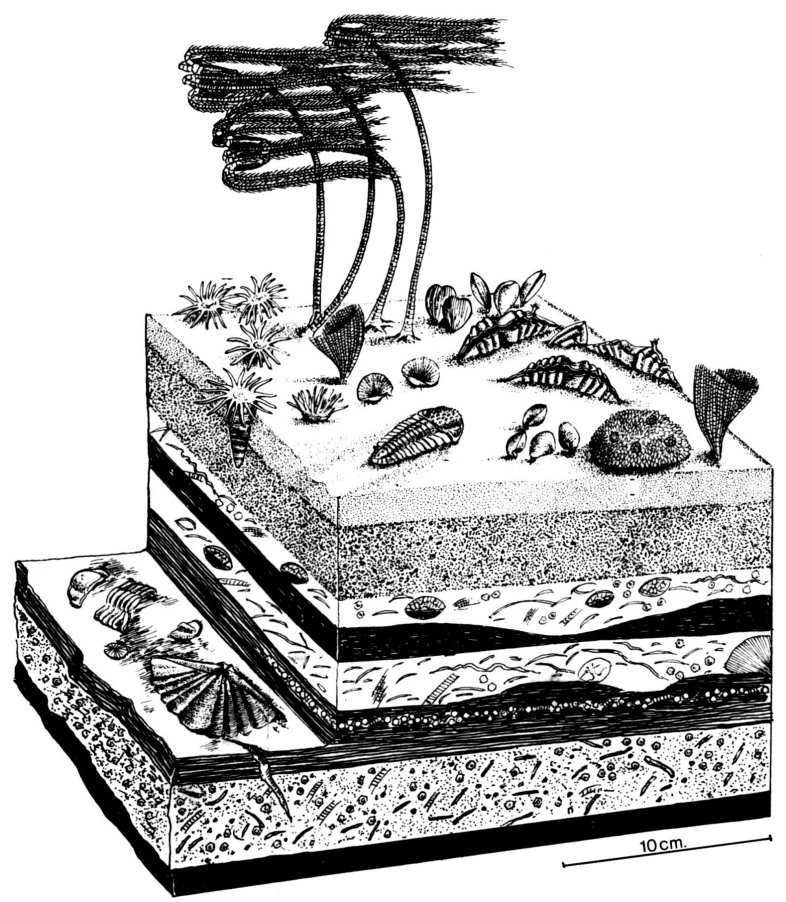

Figura 10. Comunidade do Devoniano Médio do sudoeste da Inglaterra, mostrando um trilobita epibentônico com morfotipo semelhante a "Palpebrops" goeldi (modificado de McKerrow, 1981).

Figure 10. Midle Devonian community from southwest of England showing epibenthic trilobite with a morphology comparable to " $P$ ". goeldi (modified from McKerrow, 1981). dimento e conseqüentemente o atrito, dificultando uma escavação rápida como no morfotipo I. Devido à semelhança com $P$. rana, é provável que as espécies do morfotipo III teriam a capacidade de se enrolar, embora, como nas espécies dos outros morfotipos, não tenha sido identificado nenhum espécime enrolado.

\section{CONCLUSÕES}

Todos os três morfotipos apresentam características de hábito alimentar predador/necrófago. Seriam epibentônicos, com os morfotipos I e II apresentando diferentes graus de adaptação para escavação. A presença de meio-anéis de articulação bem desenvolvidos em Elderedgeia paituna aponta para uma capacidade de enrolamento dessa espécie. As espécies do morfotipo III, "Palpebrops" goeldi e "Malvinella" australis, provavelmente poderiam ingerir uma quantidade de comida maior que os trilobitas dos morfotipos I e II, sendo predadores mais ávidos.

\section{AGRADECIMENTOS}

Ao Conselho Nacional de Pesquisa e Desenvolvimento (CNPq) pela bolsa de doutorado concedida (CFS); ao Instituto Virtual de Paleontologia-RJ/Fundação de Amparo à Pesquisa do Estado do Rio de Janeiro pelo auxílio concedido (VMMF); a Antonio Carlos Sequeira Fernandes (MN/UFRJ) pelas fotografias; a Rita de Cassia T. Cassab (MCTer/ DNPM/RJ) e a Ismar de S. Carvalho (IGEO/UFRJ) pelo empréstimo de material. A José Henrique Gonçalves de Melo (Petrobrás/CENPES) pela cedência dos dados de localização dos afloramentos fossilíferos da Formação Maecuru. A Hector R. Barrueto pela revisão do abstract. A Maurício L. Domingues pela foto da Figura 9. A Sônia Maria D. Agostinho (OFPE) pela amostra fotografada na Figura 7.

\section{REFERÊNCIAS}

Adrain, J.M. \& Westrop, S.R. 1999. Trilobite paleobiology: past, present and future. Journal of Paleontology, 73(2):161-163.

Batt, R.J. 1995. A test of new technique illustrating faunal dominance trends: application to the "Trilobite Beds" interval of the Middle Devonian Wanakah Shale in western New York. Lethaia, 28:246-258.

Branisa, L. 1965. Los fossiles guias de Bolivia. I Paleozoico. La Paz, Servicio Geologico de Bolivia, 282 p. (Boletin 6).

Brito, I.M.; Carvalho, I.S.; Vicalvi, M.A. \& Carvalho, M.G.P. 2000. Artrópodes. In: I. S. Carvalho (ed.) Paleontologia, Editora Interciência, p. 423-451.

Clarke, J.M. 1895. As trilobitas do grez de Ereré e Maecurú. Archivos do Museu Nacional, 9:1-58.

Clarkson, E.N.K. 1998. Invertebrate palaeontology and evolution. $4^{\mathrm{a}}$ ed., Cambridge, Blackwell Science, $452 \mathrm{p}$.

Cunha, R.C.P.; Gonzaga, G.F.; Coutinho, F.C.L. \& Feijó, J.F. 1994. Bacia do Amazonas. Boletim de Geociências da Petrobrás, 8(1):47-55. 
Domingues, M.L. 2003. Efeito das variações de salinidade na taxa de sobrevivência de Sphaeroma serratum (Fabricius, 1787) encontrados na Região da Marina da Glória, Rio de Janeiro, Brasil. Escola de Ciências Biológicas, Universidade do Rio de Janeiro, Monografia de Graduação, 73 p.

Eldredge, N. \& Ormiston, A.R. 1979. Biogeography of Silurian and Devonian trilobites of the Malvinokaffric Realm. In: J. Gray \& A. J. Boucot. (eds.), Historical Biogeography, Plate Tectonics and the Changing Environment, State University Press, p.147-167.

Fonseca, V.M.M. 2001. Brachiopoda (Stropheodontoidea, Chonetoidea e Delthyiridoidea) do Devoniano Médio das bacias do Amazonas e Parnaíba. Programa de Pós-Graduação em Geologia, Universidade Federal do Rio de Janeiro, Tese de Doutorado, $167 \mathrm{p}$.

Fortey, R.A. \& Owens, R.A.1999. Feeding habits in trilobitas. Palaeontology, 42(3):429-465.

Gon III, M.S. 2003. A guide to the orders of trilobites [on line]. Disponível: <http: \www.aloha.net/ smgon/triloclass.htm> [acessado em: 25 nov. 2003].

Guilardi, R.P. 2004. Tafonomia comparada e paleoecologia dos macroinvertebrados (ênfase em Trilobites), da Formação Ponta Grossa (Devoniano, sub-bacia de Apucarana), Estado do Paraná, Brasil. Instituto de Geociências, Universidade de São Paulo, Tese de Doutoramento, $113 \mathrm{p}$.

Guimarães, D. 1936. Relatório da Diretoria do Serviço de Fomento da Produção Mineral 1933-1934. Rio de Janeiro, Departamento Nacional da Produção Mineral, Serviço de Fomento da Produção Mineral, 118 p. (Boletim 14).

Harrington, H.J. 1959. General description of trilobita. In: R.C. Moore (ed.). Treatise on Invertebrate Paleontology. Lawrence, The University of Kansas Press, Arthropoda 1, p. 38-117.

Hartt, C.F. \& Rathbun, R. 1875. Devonian trilobites and mollusks of Ereré, Province of Pará, Brazil. Annals of the Lyceum of Natural History, 11:110-127.

Hughes, N.C. \& Cooper, D.L.1999. Paleobiologic and taphonomic aspects of the "Granulosa" Trilobite Cluster, Kope Formation (Upper Ordovician, Cincinati Region). Journal of Paleontology, 73(2):306-319.

Jensen, S. 1990. Predation by Early Cambrian trilobites on infaunal worms - evidence from Swedish Mickwitzia Sandstone. Lethaia, 23:29-42.

Katzer, F. 1903. Grundzüge der geologie des unteren Amazonasgebietes (des Staates Pará in Brazilien). Leipzig, Max Weg, 298 p.

Kesling, R.V. \& Chilman, R.B. 1975. Strata and megafossils of the Middle Devonian Silica Formation. Papers on Paleontology, 8:1-408.

Lieberman, S.B. 1993. Systematics and biogeography of the "Metacryphaeus group", Calmoniidae (Trilobita, Devonian), with comments on adaptative radiations and geological history of the
Malvinokaffric Realm. Journal of Paleontology, 67(4):549-570.

Lieberman, B.S.; Edgecombe, G.D. \& Eldredge, N. 1991. Systematics and biogeography of the "Malvinella group", Calmoniidae (Trilobita, Devonian). Journal of Paleontology, 65:824 843.

Loboziac, S. \& Melo, J.H.G. 2002. Devonian miospores successions of Western Gondwana: update and correlation with Southern Euroamerican miospores zones. Review of Paleobotany and Palynology, 121:133-148.

Machado, D.M.C. 1990. Bivalvia (Mollusca) do Devoniano do Amazonas (Formações Maecuru e Ererê); considerações sistemáticas e paleoecológicas. Programa de Pós-Graduação em Geologia, Universidade Federal do Rio de Janeiro, Dissertação de Mestrado, $228 \mathrm{p}$.

Machado, D.M.C.; Fonseca, V.M.M. \& Rêgo, L.V.M. 1996. Estudos preliminares sobre a distribuição espacial da macrofauna da Formação Maecuru (Devoniano Médio) da Bacia do Amazonas, Estado do Pará. In: SIMPÓSIO SUL AMERICANO DO SILURO-DEVONIANO (ESTRATIGRAFIA E PALEONTOLOGIA), 1, 1996. Anais, Ponta Grossa, UEPG, p. 239-245.

McKerrow, W.S. 1981. The ecology of fossils. Massachusetts, MIT Press, 384 p.

Melo, J.H.G. 1988. The Malvikaffric Realm in the Devonian of Brazil. In: INTERNATIONAL SYMPOSIUM ON THE DEVONIAN SYSTEM, 2, 1988. Canadian Society of Petroleum Geologists Memory, 14:669-802.

Melo, J.H.G. \& Loboziak, S. 2001. New miospore zonation of Devonian - Early Carboniferous strata in the Amazon Basin: A preliminary account. In: J.H.G. Melo \& G.J.S. Terra. (eds.), Correlação de seqüências paleozóicas sul-americanas. Ciência-Técnica-Petróleo (Seção: Exploração de Petróleo), 20:99-107.

Oliveira, A.I. \& Barbosa, O. 1940. Relatório da Diretoria, Janeiro a Setembro de 1938. Rio de Janeiro, Departamento Nacional da Produção Mineral, Divisão de Fomento da Produção Mineral, p.5-58. (Boletim 41).

Oliveira, A.I. \& Leonardos, O.H. 1978. Geologia do Brasil. $3^{\mathrm{a}}$ ed. Mossoró, Escola Superior de Agricultura de Mossoró, 813 p.

Silva, C.F. 2001. Estudo dos hábitos de vida dos macrofósseis da Formação Cabeças (Devoniano) da Bacia do Parnaíba. Escola de Ciências Biológicas, Universidade do Rio de Janeiro, Monografia de Graduação, 71 p.

Whittington, H.B.1993. Morphology, anatomy and habits of the Silurian homalonotid trilobite Trimerus. Memoirs of the Association of Australasian Palaeontologists, 15:69-83.

Received June, 2004; accepted January, 2005 